\title{
Swinging From The Hip: Use of Dynamic Motion Optimization in the Design of Robotic Gait Rehabilitation
}

\author{
Chia-Yu E. Wang, James E. Bobrow and David J. Reinkensmeyer \\ Department of Mechanical and Aerospace Engineering \\ University of California, Irvine, CA 92697
}

\begin{abstract}
In this paper we examine a method to control the stepping motion of a paralyzed person suspended on a treadmill using a robot attached to the torso and hips. A leg swing motion is created by moving the hips without contact with the legs. The problem is formulated as an optimal control problem for an underactuated articulated chain. The optimal control problem is converted into a discrete parameter optimization and an efficient gradient-based algorithm is used to solve it. Motion capture data from a human subject is compared to the results from the dynamic motion optimization. Our results indicate that it is possible for the robot to create a gait for the paralyzed person that is close to that of an unimpaired subject.
\end{abstract}

\section{Introduction}

In the U.S. alone, over 700,000 people experience a stroke each year, and over 10,000 people experience a traumatic spinal cord injury. Impairment in walking ability after such neurologic injuries is common. Recently, a new approach to locomotion rehabilitation called body weight supported (BWS) training has shown promise in improving locomotion after stroke and spinal cord injury [1]. The technique involves suspending the patient in a harness above a treadmill in order to partially relieve the weight of the body, and manually assisting the legs and hips in moving in a walking pattern (Figure 1). Patients who receive this therapy can significantly increase their independent walking ability and overground walking speed [2]. It is hypothesized that the technique works in part by stimulating remaining force, position, and touch sensors in the legs during stepping in a repetitive manner, and that residual circuits in the nervous system learn from this sensor input to generate motor output appropriate for stepping.

Clinical access to BWS training is currently limited

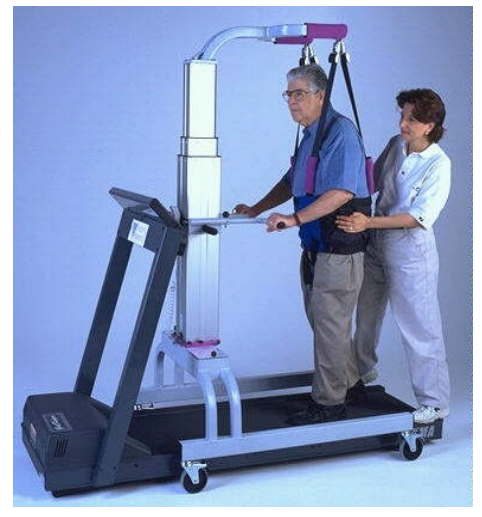

Figure 1: Partial weight bearing gait therapy device LITEGAIT I by Mobility Research, Inc.

because the training is labor intensive. Multiple therapists are often required to control the hips and legs. Several research groups are pursuing robotic implementations of BWS training in an attempt to make the training less labor intensive, more consistent, and more widely accessible $[3,4,5]$. Implementing BWS training with robotics is also attractive because it could improve experimental control over the training, thus providing a means to better understand and optimize its effects.

A difficulty in automating BWS training is that the required patterns of forces at the hips and legs are unknown. For example, the relative importance of assisting at the hip and leg is unclear. One approach toward determining the required forces is to instrument the therapists' hands with force and position transducers [3]. However, therapists are relatively limited in the forces that they can apply compared to robots, and there is no guarantee that any given therapist has selected an optimal solution.

In this paper we explore an alternate approach toward generating strategies for assisting in gait training: dynamic motion optimization. Dynamic mo- 
tion optimization provides a formalized method for determining motions for underconstrained tasks, and may reveal novel strategies for achieving the tasks. It has been used with success to simulate human control over such activities as diving, jumping, and walking $[6,7,8]$. The objective of this study was to use dynamic motion optimization to determine whether repetitive stepping by a paralyzed patient can theoretically be generated by assisting in hip motion alone.

\section{Methods}

\subsection{Human Model and Walking Mo- tion}

The human model used in this paper is similar to the one developed by Hodgins [7]. For studying the motion of the legs, the head, torso, pelvis, and arms were combined into a single rigid body. The mass of the lower leg, foot, and toes were also combined. The walking gait cycle was assumed to be bilaterally symmetric; that is, the left-side stance and swing phases were assumed to be identical to the right-side stance and swing phases, respectively. Based on this assumption only one-half of the gait cycle was simulated. We refer to the hip joint on the side of the stance phase as the stance joint and the joint on the side of the swing phase as the swing joint. For simplicity both the stance and swing hips were modeled as 2 degrees of freedom (dof) universal joints. The stance hip was allowed to rotate about axes oriented in the $x$ - and $y$ directions (refer to Figure 2). These were the dof assumed to be controlled by the hip swinging robot. The torso was assumed to remain at a fixed angle about the $z$ - axis. The swing hip was assumed to rotate only about axes in the $x$ - (i.e. leg adduction/abduction) and $z$-(i.e. hip flexion/extension). Rotation about the $y$ - axis (i.e. leg internal/external rotation) was not included in the simulation. The knee was modeled as a 1 dof hinge joint about the $z$ - axis (knee flexion/extension).

Motion capture data of key body segments for an unimpaired subject was obtained using a video-based system (Motion Analysis Corp.) Inverse kinematics was performed on the collected kinematic data in order to determine the joint positions and orientations. The height and weight of the subject were close to that used in Hodgins's human model $(180 \mathrm{~cm}$ and $85 \mathrm{~kg}$ ). One-half of the gait cycle (duration $0.633 \mathrm{sec}$ ) found by motion capture is shown in Figure 2.

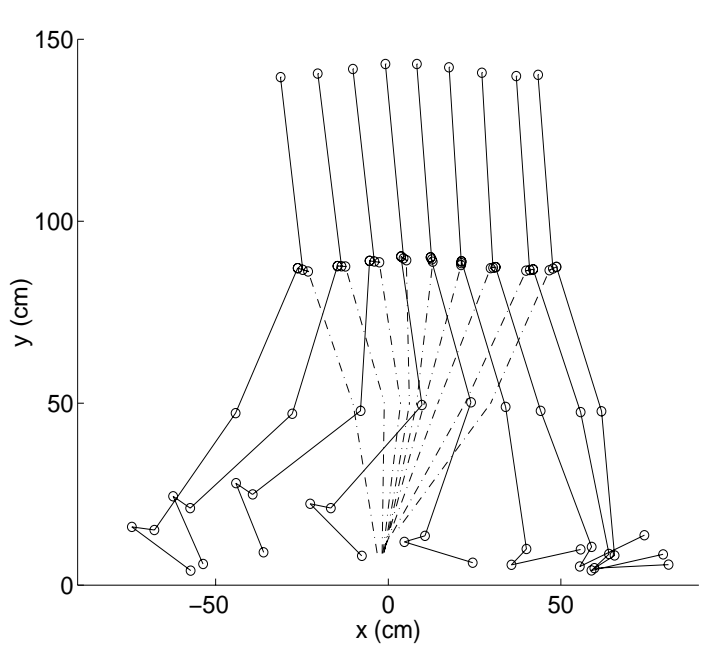

Figure 2: One-half of the gait cycle - one step.

\subsection{Formulation of the Optimal Con- trol Problem}

The objective of this study was to explore whether repetitive stepping by a paralyzed patient can theoretically be generated by moving the hip along some trajectory, without explicitly controlling the legs. That is, could a normal gait be generated by a robot that maneuvers the human torso without requiring robots that maneuver the legs? We assumed that the robot was capable of moving the stance hip along a normal, unimpaired trajectory, and simultaneously lifting the swing hip to control movement of the impaired leg. As a first approximation, we modeled the impaired leg as a flaccid (i.e. unactuated) linkage.

This problem can be addressed mathematically as an optimal control problem for an underactuated system. We are interested in obtaining a normal swing phase of the flaccid leg by shifting the hip. We used the motion of the stance hip found from the video capture data of the unimpaired subject as an input to our underactuated human model. We considered the swing motion to be an optimal control problem as follows:

$$
\begin{array}{ll}
\underset{\tau(t)}{\text { Minimize }} & J_{c}=\frac{1}{2} \int_{0}^{t_{f}} w_{e}\left(\tau_{4}^{2}+\cdots+\tau_{8}^{2}\right) d t(1) \\
\text { subject to } & H(q) \ddot{q}+h(q, \dot{q})=\tau \\
& \underline{q} \leq q \leq \bar{q} \\
& q(0)=q_{0}, \dot{q}(0)=\dot{q}_{0} \\
& q\left(t_{f}\right)=q_{f}, \dot{q}\left(t_{f}\right)=\dot{q}_{f}
\end{array}
$$

where $\tau_{1}: \tau_{3}$ are the generalized forces associated with 
the position of the stance hip (and are not included in the cost function since the position of the stance hip was specified by the motion capture data); $\tau_{4}, \tau_{5}$ are the moments corresponding to the two rotations of the stance hip (controlled by the hip-swinging robot); $\tau_{6}, \tau_{7}$ are the swing hip moments (corresponding to abduction/adduction and flexion/extension); $\tau_{8}$ corresponds to knee rotation moment; and $w_{e}$ is a weighting coefficient. $\tau_{6}, \tau_{7}$, and $\tau_{8}$ were assumed zero for the impaired leg. The joint limits, $\underline{q}$ and $\bar{q} \in \Re^{8}$, were assumed to be given. Equation (2) represents the dynamics for the human model with the joint coordinates $q \in \Re^{8}$ and the joint forces or torques $\tau \in \Re^{8}$, where $H(q)$ is the generalized mass matrix and $h(q, \dot{q})$ contains the centrifugal, Coriolis and gravitational forces and the joint friction.

In addition, it was necessary to avoid the collision of the swing leg with the stance leg and the ground. Therefore, the following penalty function, $J_{p}$, represents the collision and is added to the cost function:

$$
\begin{aligned}
J_{p 1} & =\frac{w_{p}}{2} \sum_{t_{i}=0}^{t_{f}}\left(y_{g d}-y_{f t}\left(t_{i}\right)\right)_{+}^{2}+\left(z_{l g}-z_{f t}\left(t_{i}\right)\right)_{+}^{2} \\
\phi_{+} & = \begin{cases}\phi & \text { if } \phi>0 \\
0 & \text { otherwise }\end{cases}
\end{aligned}
$$

where $(x, y, z)_{f t}$ is the Cartesian coordinate of the foot which can be computed by forward kinematics; $y_{g d}$ the height of the ground in the $y$ - direction; $z_{l g}$ the position of the stance foot in the $z$-direction; and $w_{p}$ a weighting coefficient.

The following penalty was function used to drive the passive joints of the swing leg to the desired final configuration:

$$
J_{p 2}=\frac{1}{2} \sum_{i p} w_{p 1}\left(q_{i p}\left(t_{f}\right)-q_{0 i p}\right)^{2}+w_{p 2}\left(\dot{q}_{i p}\left(t_{f}\right)-\dot{q}_{0 i p}\right)^{2}
$$

where $w_{p 1}$ and $w_{p 2}$ are the weighting coefficient.

\subsection{Dynamic Motion Optimization Via Direct Parameter Optimization}

In order to formulate the optimal control problem for a numerical solution, the joint trajectories were interpolated by uniform, $C^{4}$ continuous quintic B-spline polynomials over the knot space of an ordered time sequence.

Let the knot sequence be $0=t_{0}=\cdots=t_{5} \leq t_{6} \leq$ $\cdots \leq t_{m} \leq t_{m+1}=\cdots=t_{m+6}=t_{f}$ with $m \geq 5$ and $\Delta t=t_{j}-t_{j-1}=\frac{t f}{m-4}$ for $j=6,7, \cdots, m+1$. The joint trajectories, $q \in \Re^{8}$, are then written as

$$
q(t, P)=\sum_{j=0}^{m} p^{j} B_{j, 6}(t)
$$

where $P=\left\{p^{0}, \cdots, p^{m}\right\}$, with $p^{j} \in \Re^{8}$, are the control vertices and $B_{j, 6}$ is a quintic B-spline basis function.

For the paralyzed patient the system was underactuated with some passive joints. The dynamics of such a hybrid dynamic system consisting of both active and passive joints can be solved efficiently by the Lie group formulation in the Appendix. In order to perform the optimization, an initial trajectory was required. We guessed an initial motion and defined it with the parameter set $P$, with $q=q(t, P)$.

The optimal control problem (1) was then transformed into the following discrete parameter optimization:

$$
\begin{array}{cl}
\underset{P}{\text { Minimize }} & J_{c p}=J_{c}+J_{p 1}+J_{p 2} \\
\text { subject to } & H\left(q_{s}\right) \ddot{q}_{s}+h\left(q_{s}, \dot{q}_{s}\right)=\tau \\
& \underline{q} \leq p^{j} \leq \bar{q} \\
& p^{0}=q_{0}, p^{1}=q_{0}+\frac{1}{5} \dot{q}_{0} \Delta t \\
& p^{m}=q_{f}, p^{m-1}=q_{f}-\frac{1}{5} \dot{q}_{f} \Delta t
\end{array}
$$

Equations (12) and (13) were applied to meet the initial and final conditions (4) and (5), respectively. The actual variable parameters were $P$, excluding the fixed $p^{0}, p^{1}, p^{m-1}$, and $p^{m}$; and the total number of variable parameters was $l(m-3)$.

As described next, motions were generated by this dynamic motion optimization with the weighting coefficients $w_{e}=0.2, w_{p}=10000, w_{p 1}=6000$, and $w_{p 2}=600$. In some cases, joint torques were computed for the human model based on the estimated dynamic properties and the B-Spline joint trajectories.

\section{Results}

\subsection{Fully Actuated Model}

In order to test the optimization technique, we first studied a fully actuated human model with active hip and knee joints in the swing leg. 40 parameters ( 8 for each joint) were used in the optimization. The optimization was obtained in 15 minutes with a Pentium II $700 \mathrm{Mhz}$ PC. 
Figure 3 shows that the optimizer swings the leg in a very similar way to actual human gait. The optimization results are represented as solid lines, and the actual human data as dashed lines for comparison. The front view of the gait displays only the final configuration. The close correspondence with the human data are consistent with previous studies that suggest that human gait involves the minimization of effort/energy (e.g. [8]). Moreover, the correspondence between the optimized and actual trajectories suggests that the simulation adequately captures the essential dynamics of the human gait system. The joint angles
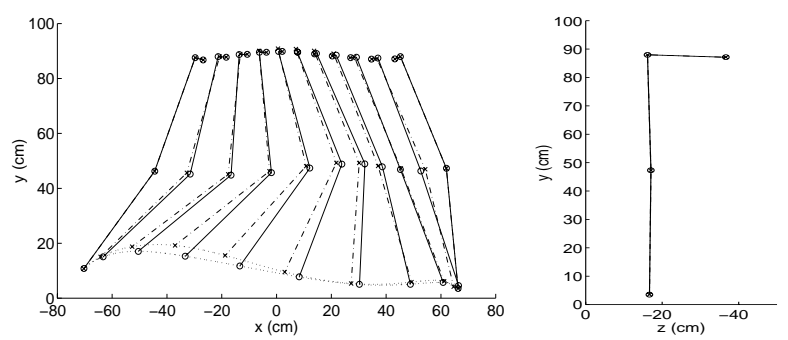

Figure 3: Fully actuated system.

and torques of the stance hip are shown in Figure 4 and 5, respectively. An interesting result was that the optimizer applied smoother joint torques (solid lines) to accomplish the step than the human did (dashed lines).
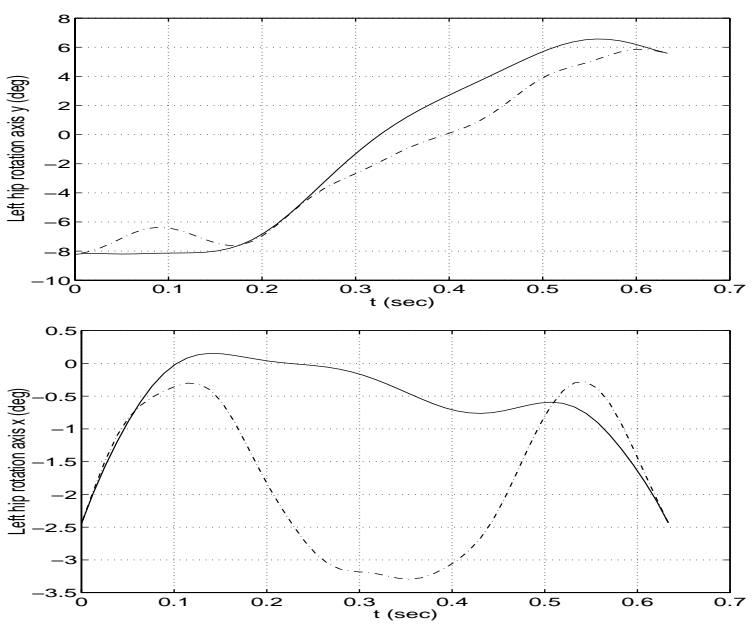

Figure 4: Stance hip angles of fully actuated system.
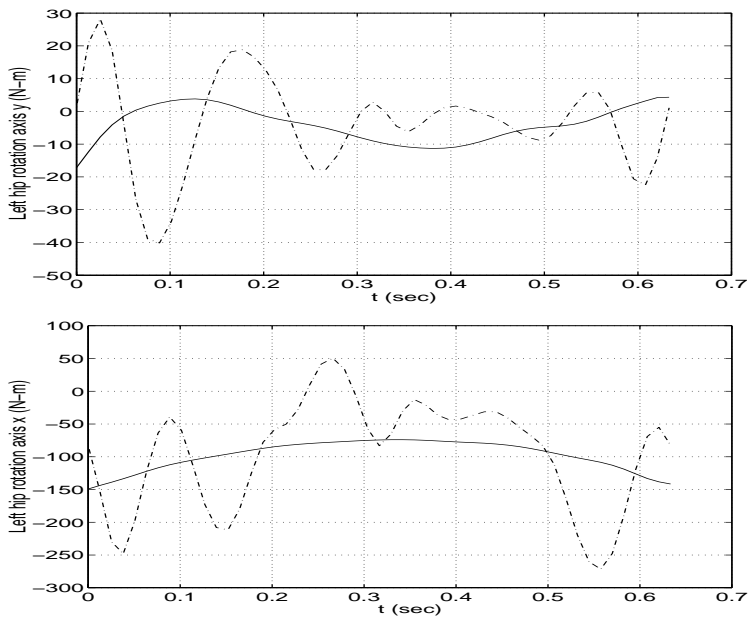

Figure 5: Stance hip torques of fully actuated system.

\subsection{Under-Actuated Model}

To simulate a paralyzed patient, the swing hip and knee joints were made passive. 22 parameters (11 for each) were used in the optimization. The optimization took approximately one hour to complete. This time was longer than the fully actuated model because of the inclusion of an additional spring-damper subsystem in the knee joint to avoid hyper-extended motion of the lower leg. The resulting motion for the underactuated system is shown in Figure 6 and its corresponding stance hip angles are shown in Figure 7. The optimizer twisted the pelvis to pump energy into the paralyzed leg, and achieved a final configuration of the leg close to the desired final configuration. Thus the optimizer was able to determine a strategy that could achieve repetitive stepping by shifting the pelvis alone. Note the large stance hip torques required to achieve the desired motion (Figure 8). The $200 \mathrm{Nm}$ torque applied about the $x$ axis rotation corresponds to a force couple with approximately $100 \mathrm{KgF}$ applied to each hip. Note also that the strategy tended to hyperextend the knee.

\section{Conclusion}

These results demonstrate the merit of investigating a hip-swinging robot for incorporation in body-weight supported locomotion training. Although it may not be possible to fully control swing from the hip, a surprising amount of control is possible, and the level of control appears sufficient for achieving repetitive step- 

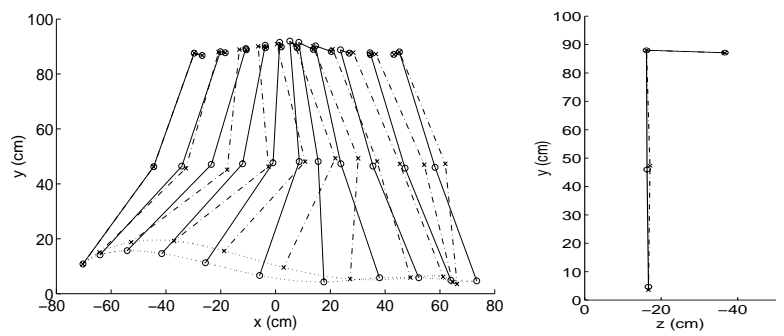

Figure 6: Under-actuated system - passive swing hip and knee $(0.633 \mathrm{sec})$.
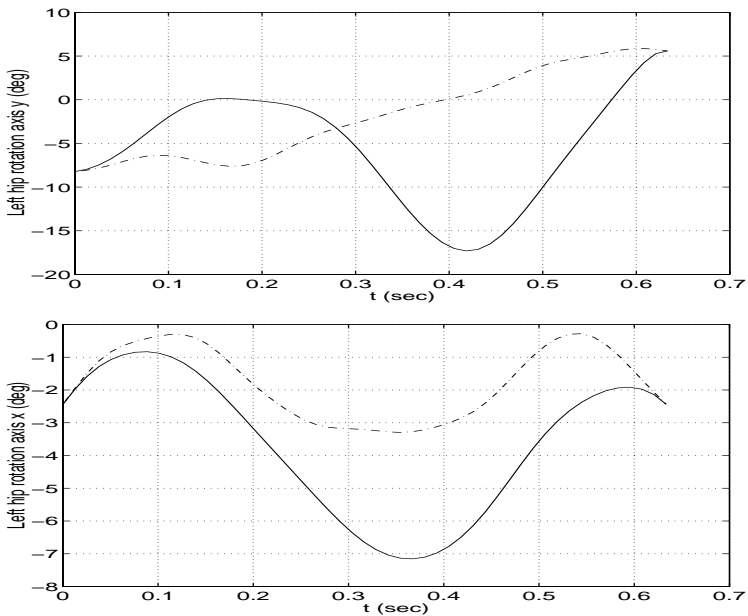

Figure 7: Stance hip angles of under-actuated system - passive swing hip and knee (0.633 sec).

ping by a paralyzed person. One caveat is that the strategy caused hyperextension of the knee. It may be possible to reduce this hyperextension by penalizing it in the cost function.

A hip swinging robot could also be useful for loading the stance leg by pressing downward on the stance hip, thus providing load-related sensory input required for stepping at the same time as assisting in swing. An important goal for future research is to determine how to couple force control of the stance leg with motion control of the swing leg.

More generally, dynamic motion optimization provides a useful tool for investigating novel strategies for assisting in locomotion rehabilitation. Finding strategies by observation of therapists is also desirable, but may miss some valuable strategies because therapists are limited in control relative to robots. For exam-
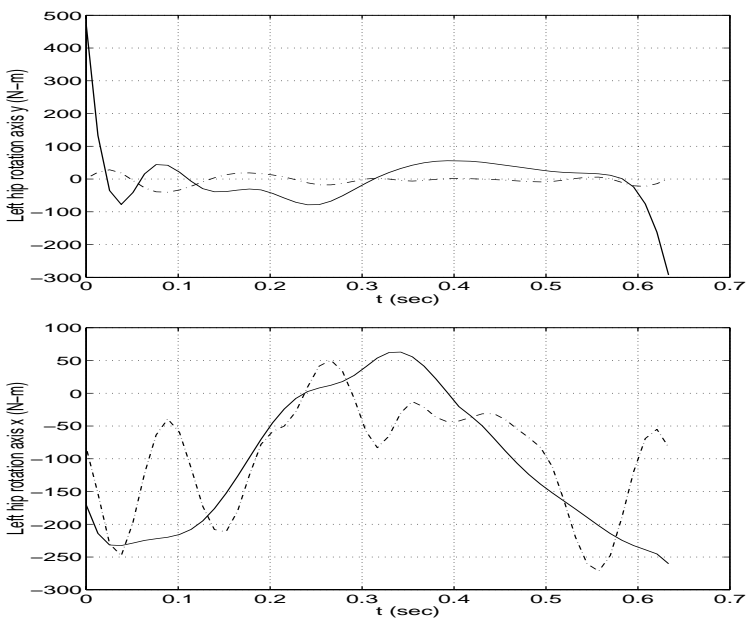

Figure 8: Stance hip torques of under-actuated system.

ple, the strategy found here requires hip torques that are quite large compared to a therapist's strength. Dynamic motion optimization also provides a formal means to automatically generate strategies on a patient-by-patient basis. One can imagine including patient-specific passive joint and reflex properties in the simulation. Also, if the patient begins to recover control over some muscles, it would be possible to model this activation and include it in the simulation. As the patient recovers walking ability, one can imagine progressing from unactuated, to partially actuated, to fully actuated simulations, with the optimization algorithm automatically determining the appropriate assistance strategy for each recovery state.

\section{Acknowledgments}

Thanks to Dana Craig, B.S., B.M.M.E., of the Department of Veterans Affairs, Long Beach Healthcare System Prosthetics Gait Lab for assistance with the video capture data.

\section{Appendix: Hybrid Dynamics}

An efficient Lie group formulation given below supplies the solution to the hybrid dynamics problem [10]. It has been implemented in the software package - Computer Simulation Tool for the Optimization of Robot Motions (CSTORM) - in our Robotics Lab.

Algorithm 1 The articulated-body recursive formu- 
lation of hybrid dynamics is:

- Initialization

$$
\begin{gathered}
V_{0}, \dot{V}_{0}, F_{n+1}, \\
F_{b n+1}=F_{n+1}, \hat{J}_{n+1}=0, J_{b n+1}=0, b_{n+1}=0
\end{gathered}
$$

- Forward recursion: $i=1$ to $n$

$$
V_{i}=A d_{T_{i-1, i}^{-1}} V_{i-1}+S_{i} \dot{q}_{i}
$$

- Backward recursion: $i=n$ to 1

$$
\begin{aligned}
\hat{J}_{i}= & J_{i}+\left[A d_{T_{i, i+1}^{-1}}^{*}\right] J_{b i+1}\left[A d_{T_{i, i+1}^{-1}}\right] \\
\hat{F}_{i}= & -a d_{V_{i}}^{*} J_{i} V_{i}+ \\
& A d_{T_{i, i+1}^{*-1}}^{*}\left(F_{b i+1}+\hat{J}_{i+1} S_{i+1} b_{i+1}\right) \\
F_{b i}= & \hat{F}_{i}+\hat{J}_{i} a d_{V_{i}} S_{i} \dot{q}_{i} \\
J_{b i}= & \begin{cases}\hat{J}_{i} & i \in I^{a} \\
\hat{J}_{i}-\hat{J}_{i} S_{i} S_{i}^{T} \hat{J}_{i} /\left(S_{i}^{T} \hat{J}_{i} S_{i}\right) & i \in I^{p}\end{cases} \\
b_{i}= & \begin{cases}\ddot{q}_{i} & i \in I^{a} \\
\left(\tau_{i}-S_{i}^{T} F_{b i}\right) /\left(S_{i}^{T} \hat{J}_{i} S_{i}\right) & i \in I^{p}\end{cases}
\end{aligned}
$$

- Forward recursion: $i=1$ to $n$

$$
\begin{aligned}
i \in I^{a} & : \\
\tau_{i} & =S_{i}^{T}\left(F_{b i}+\hat{J}_{i} A d_{T_{i-1, i}^{-1}} \dot{V}_{i-1}+\hat{J}_{i} S_{i} b_{i}\right) \\
i \in I^{p} & : \\
\ddot{q}_{i} & =b_{i}-S_{i}^{T} \hat{J}_{i} A d_{T_{i-1, i}^{-1}} \dot{V}_{i-1} /\left(S_{i}^{T} \hat{J}_{i} S_{i}\right) \\
\dot{V}_{i} & =A d_{T_{i-1, i}^{-1}} \dot{V}_{i-1}+S_{i} \ddot{q}_{i}+a d_{V_{i}} S_{i} \dot{q}_{i}
\end{aligned}
$$

where the index $i$ indicates that the correspondence is represented in the $i^{\text {th }}$ link frame counted from the base frame $(i=0)$. The joint screw, spatial velocity, spatial acceleration and spatial force are written as $S$, $V, \dot{V}$, and $F \in s e(3)$, respectively. Particularly, $V_{0}$ and $\dot{V}_{0}$ represent the spatial velocity and acceleration of the base, respectively, while $F_{n+1}$ represents the external spatial force. $T_{i-1, i} \in S E(3)$ denotes the transformation from the $i-1^{\text {th }}$ link frame to the $i^{\text {th }}$ link frame. The joint velocity, acceleration, force and the Coulomb and viscous friction are written as $\dot{q}, \ddot{q}, \tau, f_{c}$ and $f_{v} \in \Re$, respectively. And $J$ is the spatial inertia matrix. The set of active joints in which the forward dynamics is applied is represented as $I^{a}$; and the set of passive joints in which the inverse dynamics is applied is represented as $I^{p}$. The detailed description of notation and operators can be found in [10].

\section{References}

[1] I. Wickelgren, "Teaching the spinal cord to walk," Science, Vol. 279 pp. 319-321, 1998.

[2] H. Barbeau, K. Norman, J. Fung. M. Visintin, M. Ladoucer, "Does neurorehabilitation play a role in the recovery of walking in neurological populations," Annals New York Academy of Sciences, Vol. 860, pp. 377-392, 1998.

[3] A. Bejczy, "Towards development of robotic aid for rehabilitation of locomotion-impaired subjects" Proc. 1st Workshop Robot Motion and Control (RoMoCo'99), pp. 9-16, 1999.

[4] S. Hesse, and D. Uhlenbrock, "Gait pattern of severely disabled hemiparetic subjects on a new controlled gait trainer as compared to assisted treadmill walking with partial body weight support" Clinical Rehabilitation, vol. 13, pp. 401-10, 1999.

[5] D.J. Reinkensmeyer, W.K. Timoszyk, R.D. de Leon RD, R. Joynes, E. Kwak, K. Minakata, V.R. Edgerton, "A robotic stepper for retraining locomotion in spinal-injured rodents., San Francisco, California, April" Proc 2000 IEEE International Conference on Robotics and Automation, pp. 28892894, 1999.

[6] J. V. Albro, G. A. Sohl, J. E. Bobrow, and F. C. Park, "On the computation of optimal high-dives," IEEE International Conference on Robotics and Automation, Vol. 4 pp. 3958-3963, 2000.

[7] J. K. Hodgins, "Three-dimensional human running," IEEE International Conference on Robotics and Automation, Vol. 4 pp. 3271-3276, 1996.

[8] M. G. Pandy and F. C. Anderson, "Dynamic simulation of human movement using large-scale models of the body," IEEE International Conference on Robotics and Automation, Vol. 1 pp. 676-681, 2000 .

[9] C-Y. E. Wang, W. K. Timoszyk, and J. E. Bobrow, "Weightlifting motion planning for a Puma 762 robot," IEEE International Conference on Robotics and Automation, Vol. 1 pp. 480-485, 1999.

[10] C-Y. E. Wang, Optimal Path Generation For Robots, Ph.D. Thesis, University of California, Irvine, 2000. 